\title{
MILLIMETER WAVE PATCH ANTENNA ARRAY FOR 5G WIRELESS COMMUNICATION SYSTEMS
}

\author{
A.B. Gnilenko ${ }^{1 *}$, S.V. Plaksin ${ }^{2}$ \\ ${ }^{\text {I}}$ Oles Honchar Dnipro National University, Dnipro, Ukraine \\ ${ }^{2}$ Institute of Transport Systems and Technologies of NAS of Ukraine, Dnipro, Ukraine \\ *e-mail:gnilenko@ua.fm
}

\begin{abstract}
Millimeter waves are now considered as an important part of 5G spectrum. Higher frequencies provide larger bandwidth giving the ability to support very high data rate, ultra high capacity and very low latency. The utilization of millimeter wave frequency bands for 5G mobile applications requires effective solutions in the design of antennas and antenna arrays which are the key parts of modern communication systems. In this paper a $4 \times 4$ microstrip patch antenna array sub-module is presented to be a part of $5 \mathrm{G}$ wireless communication system. The antenna array is designed and optimized to operate at a frequency of $85 \mathrm{GHz}$ which corresponds to the middle of the second atmospheric transparency window. The antenna array is simulated using the time domain solver of the CST Microwave Studio software package. Simulation results are demonstrated and discussed for an optimized array. The designed patch antenna array provides good directivity characteristics with a main lobe magnitude of $16 \mathrm{dBi}$, angular width of 28 degree and can be applied as a part of a wireless communication system operating at a high frequency band of $5 \mathrm{G}$ frequency range.

Keywords: microstrip antenna, antenna array, millimeter waves, $5 \mathrm{G}$, directivity pattern.
\end{abstract}

Received 09.11.2019; Received in revised form 10.12.2019; Accepted 23.12.2019

\section{Introduction}

Most modern wireless communication systems operate at frequencies below $6 \mathrm{GHz}$. But the commonly used frequency bands no longer cope with the increasing data flow transmitted in real time due to the lack of wide enough bandwidth for those operating frequencies. The evident solution is to use higher operating frequencies for which broad frequency bands can be easily obtained. That is why the millimeter waves attract growing attention as a spectrum resource which can provide very large bandwidths with ultra-high capacity and very low latency [1].

Moving to millimeter wave bands for $5 \mathrm{G}$ applications requires new technologies and designs of antennas for wireless communication systems which can be capable of dynamically steering and precisely forming the radio beam between mobile-station and basestation systems. In order to obtain an efficient beam-steerable antenna system for 5G applications, the smaller antennas arranged as an array can be employed. The best choice for these demands is microstrip patch antennas [2,3]. Due to compact size, low profile, light weight, low cost and great conformity microstrip patch antennas and antenna arrays are widely used in various ground and satellite communication systems, cellural communication systems, transceivers, remote sensing and global position systems. That is why patch antennas and antenna arrays have gained a lot of interest in the past years for millimeter wave applications.

Due to higher losses at millimeter wave frequencies than at lower frequencies the rigorous antenna design is needed to minimize losses and achieve high radiation efficiency. In this paper we present the design of $4 \times 4$ patch antenna array optimized for an operating frequency of $85 \mathrm{GHz}$ which corresponds to the second atmospheric transparency window. The patch antenna array is supposed to be a part of $5 \mathrm{G}$ wireless communication systems.

\section{Millimeter wave bands for $5 \mathrm{G}$ applications}

Modern cellular and Wi-Fi systems suffer from spectrum shortage in the conventional microwave bands. That is why millimeter wave bands are attracting growing attention by main players in the telecommunication market. Millimeter wave bands are now considered as 
an additional spectrum bands for $5 \mathrm{G}$ cellular networks. Today's telecommunication networks utilize frequencies up to $6 \mathrm{GHz}$ for wireless data transmission. Particularly, 5G networks can use the existing LTE frequency range from $600 \mathrm{MHz}$ to $6 \mathrm{GHz}$. But future $5 \mathrm{G}$ applications will inevitably require using new spectrum bands in much higher frequency ranges up to $100 \mathrm{GHz}$ and beyond, utilizing millimeter and sub-millimeter waves [4]. Very high 5G frequency bands enables speeds up data transmission in the tens of Gbps at extremely low latency [5]. The major drawback of millimeter wave bands is very small coverage areas and poor penetration through buildings. Propagation for these frequencies often requires line-of-sight conditions between the base station and device.

However, many researchers design new technological solutions to overcome those drawbacks. As can be seen in the chart shown in Fig. 1 the advantages of millimeter wave bands in terms of high transmission data rate, high capacity and low latency can drastically overcome the shortage of coverage, especially in dense urban area, where the distances between the transmitting and receiving stations are minimal.

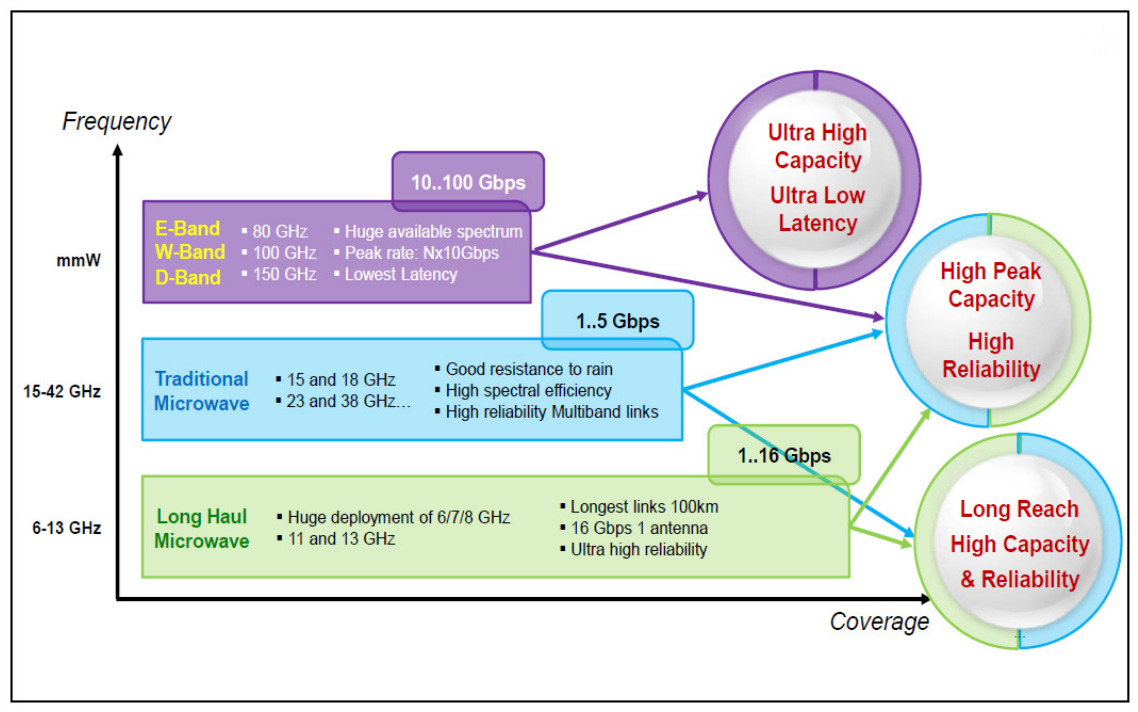

Fig. 1. 5G frequency band features [5].

The Federal Communication Commission (FCC) has licensed only the 24 and 28 $\mathrm{GHz}$ bands for $5 \mathrm{G}$ use right now. But the FCC considers auctioning further high-band spectrum as a priority and expects to auction off the 37,39 , and $47 \mathrm{GHz}$ bands for $5 \mathrm{G}$ by the end of 2019 [6]. At the same time the FCC recognizes that unlicensed spectrum will be important for $5 \mathrm{G}$, and the agency is creating new opportunities for the next generation of Wi-Fi utilizing very high-band spectrum up to $96 \mathrm{GHz}$. The very high frequency bands such as $71 \mathrm{GHz}$ to $76 \mathrm{GHz}$ and $81 \mathrm{GHz}$ to $86 \mathrm{GHz}$ are now discussed as candidate bands for 5G among key industry players in the telecommunication market [7] (see Fig. 2). However, these bands are currently at the proposal stages.

Higher frequencies are advantageous because of higher data transfer rate, wider frequency bandwidth and narrower radiation beam. The main drawback is severe atmospheric absorption as frequency increases to the range of millimeter waves. Besides the common tendency of absorption increase at high frequencies, there are local absorption maximums due to the presence of oxygen molecules in the air at $60 \mathrm{GHz}$ and $118 \mathrm{GHz}$, and water vapor at $22 \mathrm{GHz}$ and $180 \mathrm{GHz}$ [8] as can be seen in Fig 3 . 


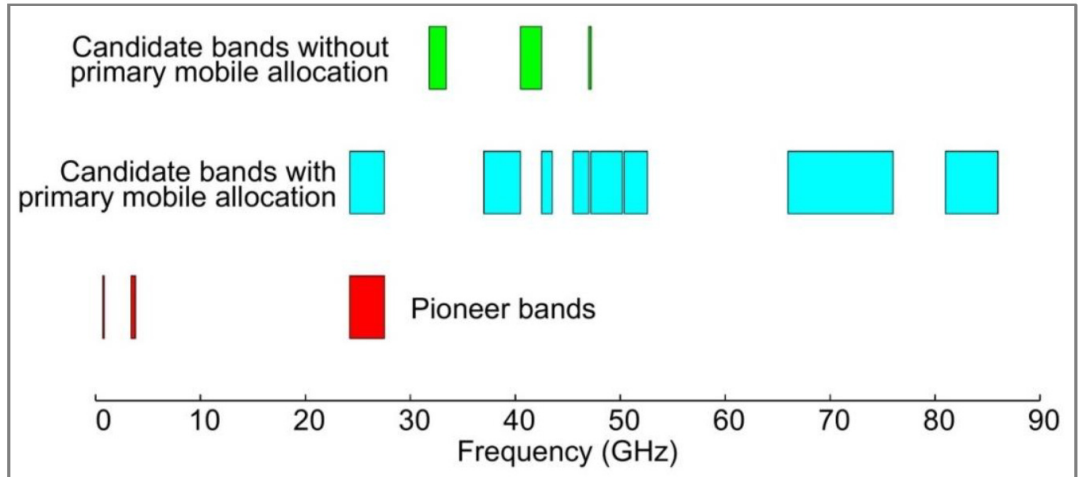

Fig. 2 Candidate frequency bands for $5 \mathrm{G}$ applications [7].

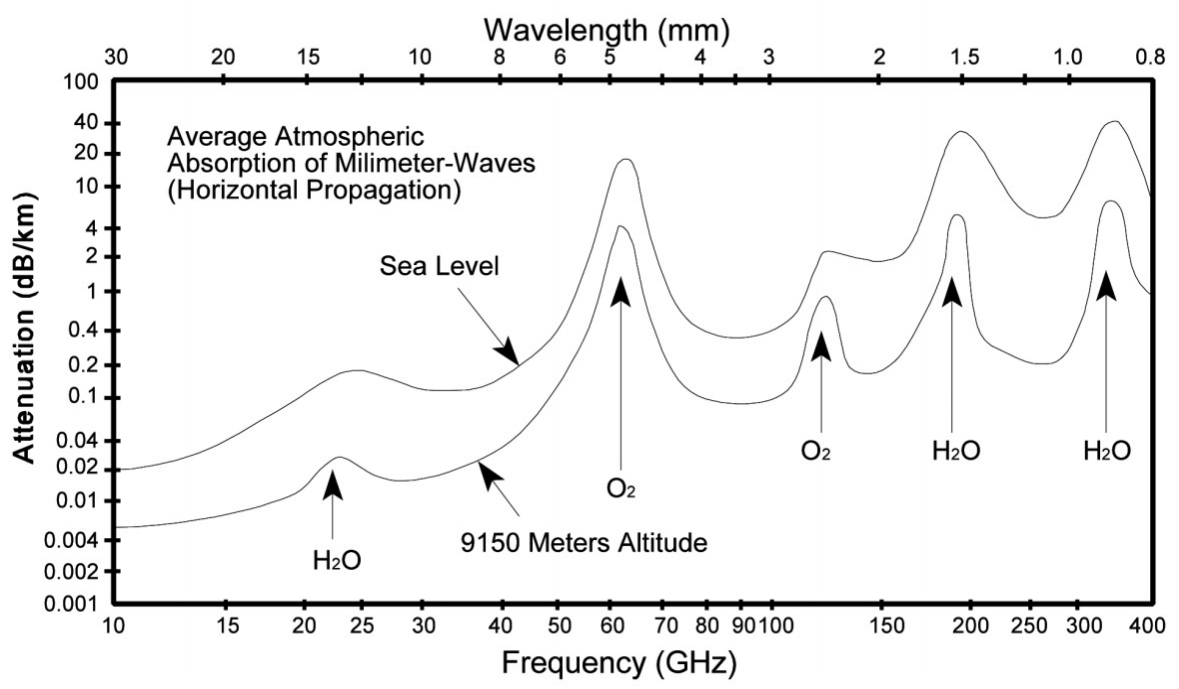

Fig. 3. Electromagnetic wave attenuation in atmosphere.

However, wireless communication and power transition may be possible with minimal losses even at high frequencies if they are located in atmospheric transparency windows between the local maxima of absorption. From this point of view the design of millimeter wave antenna array systems working at operating frequencies within the atmospheric transparency windows is of great interest.

\section{Antenna array design}

The patch antenna array is designed along with its corporate feeding circuit based on microstrip lines. The geometry of the antenna array is shown in Fig. 4. The antenna array has 16 symmetrically fed radiating patches placed on Rogers RT5870 substrate with permittivity $\varepsilon_{\mathrm{r}}=2.33$ and thickness $h=0.127 \mathrm{~mm}$. This $4 \times 4$ array is considered to be a terminal sub-module of a radiation system fed by $100 \Omega$ input microstrip line. The feed circuit with the common input is placed on the same side of the substrate with the radiating patches. The chosen array geometry is advantageous by its technological simplicity, compact size, light weight, and the high degree of conformity $[9,10]$. However, placing feeding circuit elements on the face side of the antenna array may 
cause mutual coupling, surface wave generation, and parasitic radiation which degrade antenna characteristics.

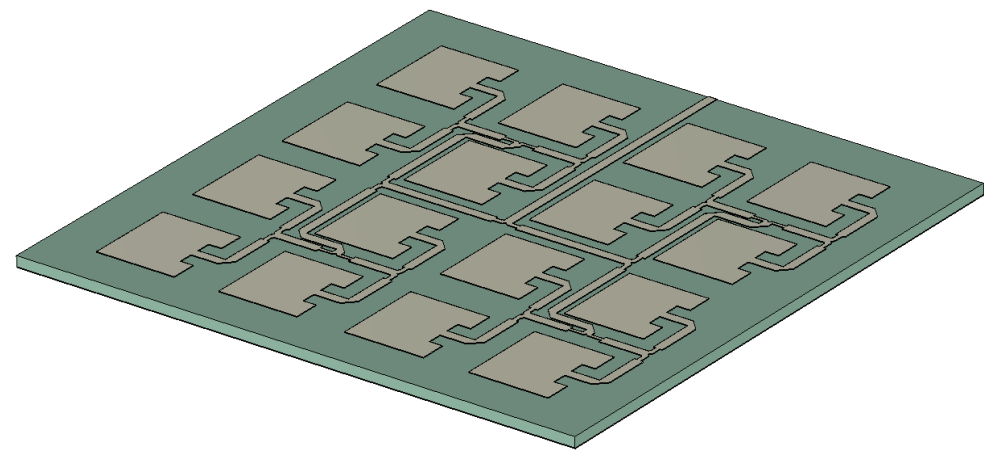

Fig. 4. The geometry of $4 \times 4$ patch antenna array.

To effectively deliver input power to the patches, impedances of all feeding circuit elements and the radiating patches have to be matched. We use mitered T-junctions with quarter wavelength microstrip line step transformers as power dividers and mitered microstrip bends for circuit packaging. For matching the impedance of feeding microstrip lines with the input impedance of the patches, the inset feeding technique is involved into the design. Parameters of the radiating patches and the feeding circuit elements are optimized for the input return loss minimum at an operating frequency of $85 \mathrm{GHz}$.

\section{Simulation results}

The antenna array has been simulated using CST Microwave Studio software package. The dependences of the reflection coefficient magnitude and total efficiency versus frequency are demonstrated in Fig. 5. It can be seen in Fig. 5(a) that the reflection coefficient reaches $-29 \mathrm{~dB}$ at the operating frequency. The impedance bandwidth at $-10 \mathrm{~dB}$ return loss level is equal to $3.5 \mathrm{GHz}$.

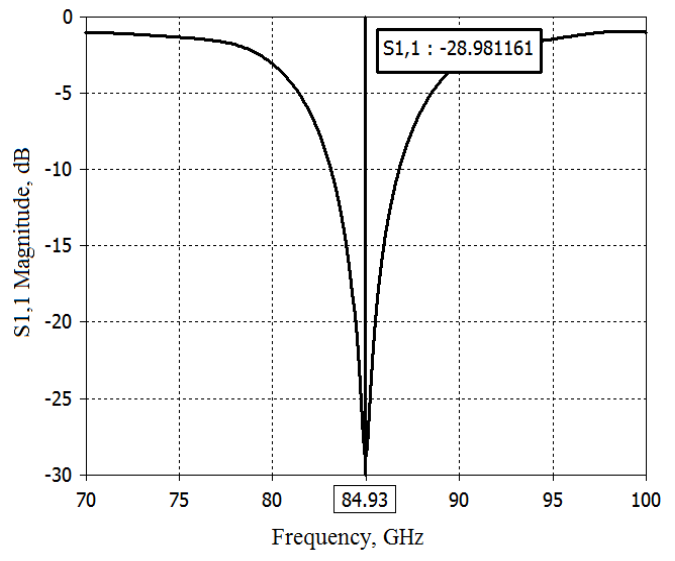

(a)

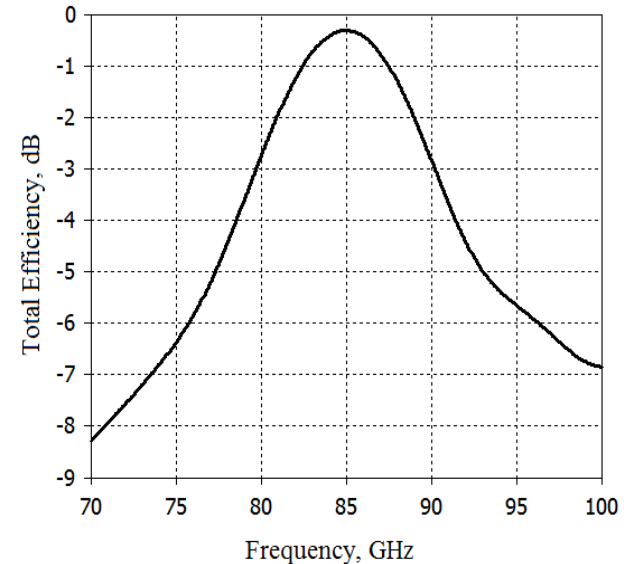

(b)

Fig. 5. Input return loss (a) and total efficiency (b) characteristics of the antenna array. 
The total efficiency shown in Fig. 5(b) is the product of the radiation efficiency and the impedance mismatch loss of an antenna connected to a feeding circuit. It can be seen that the total efficiency at an operating frequency of $85 \mathrm{GHz}$ is equal to $-0.31 \mathrm{~dB}$ or $96.5 \%$. The curve of total efficiency dependence demonstrates a resonant character with a maximum near a frequency of $85 \mathrm{GHz}$ because the circuit matching elements are tuned to that operating frequency. At a distance of the operating frequency the impedance mismatch loss rapidly increases and the total efficiency decreases.

The radiation pattern of the antenna array is shown in Fig. 6 for polar coordinates and 3D view. As can be seen in Fig. 6(a), the main lobe magnitude is $16 \mathrm{dBi}$ at a direction of 1.0 degree. The side lobe level is $-19.3 \mathrm{~dB}$. The angular width of the directivity pattern at $3 \mathrm{~dB}$ level is 28 degree.

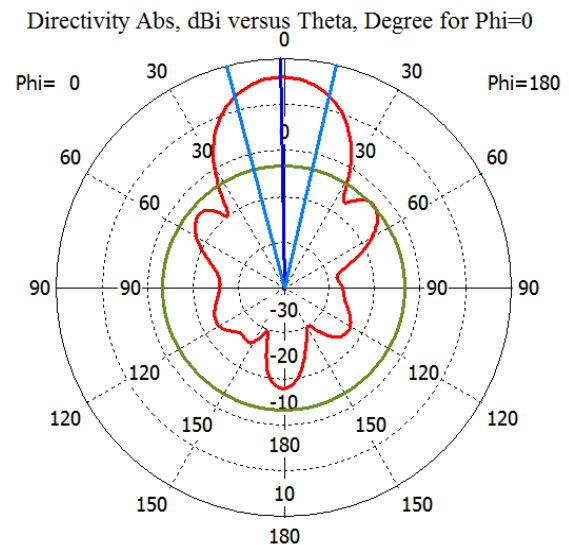

(a)

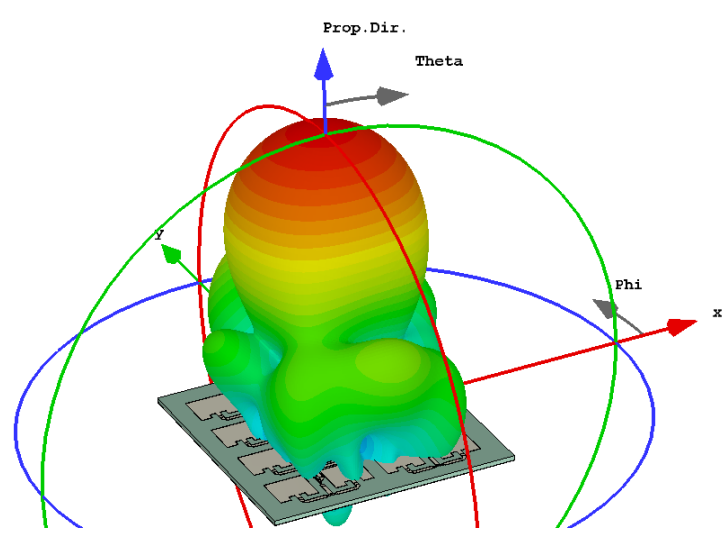

(b)

Fig. 5. Directivity pattern of the antenna array: polar plot (a) and 3D view (b).

Fig. 6(b) demonstrates 3D picture of the directivity pattern shown along with the antenna array structure. As can be seen, the side lobes are mainly produced by parasitic radiation from the microstrip discontinuities of the feeding circuit. An essential factor of side lobe appearance is the distance between the radiating patches. The greater the interpatch distance the greater the side lobes. But more compact circuit packaging may lead to parasitic interactions between neighboring feeding circuit elements and patches. That is why a trade-off should be found between an acceptable side lobe level and antenna characteristic degradation by parasitic interactions of circuit elements which arise due to more compact packaging.

\section{Conclusions}

The $4 \times 4$ microstrip patch antenna array is simulated and optimized for an operating frequency of $85 \mathrm{GHz}$. This operating frequency corresponds to the middle of the second atmospheric transparency window providing higher data transfer rate, wider frequency band, and narrower radiation beam for effective wireless communication with minimal power losses in the atmosphere. The simulation results of the antenna array show that an input return loss level is equal to $-29 \mathrm{~dB}$, the main lobe magnitude reaches $16 \mathrm{dBi}$ and the angular width of the directivity pattern is equal to 28 degree. Due to its characteristics, technological simplicity, great conformity, low profile, light weight, and low cost the proposed antenna array can be applied as a part of a wireless communication system for very-high frequency band $5 \mathrm{G}$ applications. 


\section{References}

1. Liu, D. Advanced Millimeter-Wave Technologies: Antennas, Packaging and Circuits / D. Liu, B. Gaucher, U. Pfeiffer, J. Grzyb. - John Wiley \& Sons Ltd., 2009. $827 \mathrm{p}$.

2. Balanis, C.A. Modern Antenna Handbook / C.A. Balanis. - John Wiley \& Sons Inc., 2008. $-1704 \mathrm{p}$.

3. Lee, K.F. Microstrip Patch Antennas / K.F. Lee, K.M. Luk. - London: Imperial College Press, 2011. - 524 p.

4. Ancans, G. Spectrum considerations for 5G mobile communication systems / G. Ancans, V. Bobrovs, A. Ancans, D. Kalibatiene // Procedia Computer Science. - 2017. No. 104. - P. $509-516$.

5. Lombardi, R. Microwave and millimeter-wave for 5G transport / R. Lombardi // ETSI White Paper. - 2018. - No. 25. - P. 1 - 16.

6. The FCC's 5G fast plan [Electronic resource] / Federal Communication Commission. - Retrieved September 16, 2019 from http:// www.fcc.gov/5G.

7. Spectrum for 5G / Euro-5G - Supporting the European 5G Initiative. Report D5.4. - 2017. - P. $1-18$.

8. Altshuler, E.E. A comparison of experimental and theoretical values of atmospheric absorption at the longer millimeter wavelengths / E.E. Altshuler, R.A. Marr // IEEE Trans. Antennas Propag. - 1988. - Vol. AP-36, No. 10. - P. 1471 - 1480.

9. Jobins, G. Design and optimization of $2 \times 2$ corporate-series fed microstrip antenna array / G. Jobins, B. Lethakumari // Int. Journal of Engineering \& Technology. 2018. - Vol. 7, No. 1.9. - P. $84-87$.

10. Shambavi, K. Design and analysis of high gain millimeter wave microstrip antenna array for wireless applications / K. Shambavi, C. A. Zachariah, T. N. P. Krishna // Journal of Theoretical and Applied Information Theory. - 2009. - Vol. 7, No. 2. - P. $159-164$ 